\title{
Non-triggered auroral substorms and long-period (1-4 mHz) geomagnetic and auroral luminosity pulsations in the polar cap
}

\author{
Nadezda Yagova $^{1}$, Natalia Nosikova ${ }^{1,2}$, Lisa Baddeley ${ }^{3,4,5}$, Olga Kozyreva ${ }^{1}$, Dag A. Lorentzen ${ }^{3,4,5}$, \\ Vyacheslav Pilipenko ${ }^{1,6}$, and Magnar G. Johnsen ${ }^{7}$ \\ ${ }^{1}$ Schmidt Institute of Physics of the Earth, Moscow, Russia \\ ${ }^{2}$ National Research Nuclear University "MEPhI", Moscow, Russia \\ ${ }^{3}$ University Centre in Svalbard, Svalbard, Norway \\ ${ }^{4}$ Birkeland Centre for Space Science (BCSS), University of Bergen, Bergen, Norway \\ ${ }^{5}$ British Antarctic Survey, Cambridge, UK \\ ${ }^{6}$ Space Research Institute of the Russian Academy of Sciences, Moscow, Russia \\ ${ }^{7}$ Troms $\varnothing$ Geophysical Observatory, UiT - the Arctic University of Norway, Troms $\varnothing$, Norway \\ Correspondence to: Nadezda Yagova (nyagova@yandex.ru)
}

Received: 20 June 2016 - Revised: 8 January 2017 - Accepted: 6 February 2017 - Published: 8 March 2017

\begin{abstract}
A study is undertaken into parameters of the polar auroral and geomagnetic pulsations in the frequency range 1$4 \mathrm{mHz}(\mathrm{Pc} 5 / \mathrm{Pi} 3)$ during quiet geomagnetic intervals preceding auroral substorms and non-substorm background variations. Special attention is paid to substorms that occur under parameters of the interplanetary magnetic field (IMF) conditions typical for undisturbed days ("non-triggered substorms"). The spectral parameters of pulsations observed in auroral luminosity as measured by a meridian scanning photometer (Svalbard) in the polar cap and near the polar boundary of the auroral oval are studied and compared with those for the geomagnetic pulsations measured by the magnetometer network IMAGE in the same frequency range. It is found that $\mathrm{Pc} 5 / \mathrm{Pi} 3$ power spectral density (PSD) is higher during pre-substorm time intervals than for non-substorm days and that specific variations of pulsation parameters ("substorm precursors") occur during the last 2-4 pre-substorm hours.
\end{abstract}

Keywords. Magnetospheric physics (auroral phenomena)

\section{Introduction}

Nightside auroral zone disturbances have been studied for a long time. There are three main types of disturbances (Lyons, 2000): poleward boundary intensifications (PBIs), substorms, and effects of solar wind dynamic pressure enhancements. Solar wind dynamic pressure enhancements cause an increase in global proton aurora (Laundal and Østgaard, 2008). PBIs are the most common type of disturbances which occur during all levels of geomagnetic activity (including all phases of substorms, storms, etc.) and have an auroral signature and associated ground magnetic perturbations from a few tens of nanotesla up to $500 \mathrm{nT}$. Evidence suggests that PBIs might correspond to flow bursts from the distant tail plasma sheet (Lyons, 2000).

Substorms are a far more dramatic and large-scale phenomenon, related to ground magnetic disturbances of the order of a few hundred nanotesla and auroral activity. The problem of substorm triggering is of key importance for substorm physics. Initially, it was thought that a northward turning of the interplanetary magnetic field (IMF) $B_{Z}$ component was the required or main trigger (Aubry et al., 1970; Lyons, 1995). Later it was reported (Hsu and McPherron, 2004) that "non-triggered substorms", which can be associated with a steady southward IMF $B_{Z}$ or northward IMF ("non-triggered substorms with positive IMF $B_{Z}$ "), have the same features, such as a negative bay in an auroral low (AL) index and Pi2 waveforms associated with onset, as "normal substorms". The main difference between the two is that nontriggered substorms usually have weaker magnetic field signatures. Even so, an intense double-onset substorm, which took place on 14 December 2004 under moderate parameters of the solar wind (SW) and steady northward IMF was observed (Liu et al., 2010). Liu et al. (2013) reported a unique 
substorm with an extremely long growth phase which developed under quiet IMF and solar wind conditions. For more detailed information about other types of nightside auroral zone activity, please see, for example, Henderson (2012).

Statistical studies show that solar wind parameters effect substorm onset, in particular the starting time and location of substorm onset as well as the greatest possible poleward latitude of onset (Wang et al., 2005). However, up to $40 \%$ of all substorms cannot be associated with an IMF trigger and the inclusion of IMF $B_{Y}$ as a potential trigger does not significantly change the statistics. Several authors have proposed different criteria that allow us to count some SW parameter variations as an external trigger (see Newell and Liou, 2011; Gallardo-Lacourt et al., 2012, for the detailed comparison of results depending on selection criteria). Even the existence of a minimal threshold value of any IMF or SW parameter necessary for substorm excitation is also a subject of discussion. Hence, Newell et al. (2016) concluded that any change in the SW velocity or IMF $B_{Z}$ "does increase the odds of a substorm when compared to no change". The question of internal triggers has also been discussed by various authors, (e.g., Hurricane et al., 1999). Hurricane et al. (1999) presented a model of substorm detonation. The model suggests a mechanism whereby both internally and externally triggered substorms and pseudo-breakups can be explained. Also, the model explains the very fast onset timescale and localized initial signature of onsets. In addition,possible mechanisms of storing energy input into the magnetotail for the event were discussed. The authors suggested that the energy had been stored during southward IMF, with additional energy then being supplied by the dayside reconnection related to the dominant IMF $B_{Y}$.

Different kinds of instabilities are also proposed to be an internal trigger. Panov et al. (2012) observed "strong magnetic oscillations with period from 20 to $100 \mathrm{~s}$ " in the plasma sheet a few tens of minutes before the breakup using THEMIS data. The authors concluded that these measurements partly confirm the mechanism of the development of a kinetic ballooning or interchange instability mentioned by Pritchett and Coroniti (2010). Golovchanskaya et al. (2015) assumed that Pi2 and Pc5 pulsations, associated with substorms, might be interpreted as a signature of the ballooningtype perturbations in the magnetotail. The THEMIS mission gave new possibilities for a precise experimental study of substorm development from the distant to the near tail during the last pre-onset minutes (see, e.g., Angelopoulos et al., 2008; Nishimura et al., 2010; Lui, 2011), and these studies again raise a question about particular mechanisms of internal triggering.

One of the important questions for understanding substorm generation is the possible existence of specific presubstorm variations of plasma, particles, and electromagnetic field parameters (substorms precursors). Nishimura et al. (2013) studied pre-substorm activity on the dayside polar region using $630.0 \mathrm{~nm}$ wavelength data from an all-sky im- ager (ASI) located at $82.2^{\circ} \mathrm{N}$ and $56.4^{\circ} \mathrm{W}$ corrected geomagnetic coordinates as well as the ASI array of the THEMIS mission which consists of 21 white light imagers located between Greenland and Alaska. The authors suggested that the pre-onset polar cap patches and arcs propagate from the dayside polar cap to the nightside and might lead to substorm onset.

Aurora is characterized by a complicated spectrum of magnetic field oscillations, in particular in the ultra-low frequency (ULF) range, including $\mathrm{Pi} 2$ pulsations as an onset sensor (Olson, 1999) and pulsations both of Pi1/2 and Pc4-5 types specific to different stages of a substorm (Samson et al., 1992; Holter et al., 1995; Mager and Klimushkin, 2008). Presubstorm features in ULF parameters were studied by Saito et al. (2008) and Liang et al. (2009) but only concentrating on the final pre-onset minutes. The initial idea of Samson et al. (1992) about a possible role of field line resonance (FLR) in substorm triggering was later developed by Rae et al. (2014), who found that specific resonance ULF waves with high azimuthal wave numbers can be a candidate for a trigger of the substorm onset.

The problem of a preexisting population of ULF waves at longer pre-onset times has yet not been studied in detail. Only a few studies have paid attention to ULF wave activity at approximately $1 \mathrm{~h}$ or more than $1 \mathrm{~h}$ before onset. Probably, Heacock and Chao (1980) were the first who found presubstorm growth of Pi pulsation power in the polar cap. Their study of Pi pulsations at the Thule polar cap station showed a Pi enhancement centered at $1.5 \mathrm{~h}$ before an onset at auroral latitudes. The authors classified all the substorms as isolated or non-isolated according to the auroral electrojet (AE) index during the preceding time interval and found that $\mathrm{Pi}$ enhancement at Thule appeared in both cases. Later, Yagova et al. (2000) studied Pi3 amplitudes in the polar cap using the data from the VOS station in Antarctica and found that the amplitudes were high during the final pre-substorm hours when compared to non-substorm days. Golovchanskaya et al. (2015) reported a visible precursor approximately $40 \mathrm{~min}$ before a substorm onset (the so-called east-west type precursor activity of the auroral onset). Wing et al. (2013) studied the power of wave/broadwave, monoenergetic, and diffuse auroral electrons $2 \mathrm{~h}$ before and $3 \mathrm{~h}$ after onset of an isolated substorm (i.e., a substorm which is separated by at least $5 \mathrm{~h}$ from other substorms) using data from DMSP satellites. It was reported that the wave power observed in the monoenergetic electron aurora peaked in the dusk-midnight sector and slowly started increasing about $1 \mathrm{~h}$ and $15 \mathrm{~min}$ before an onset. Possible explanations were provided related to the stretching of the magnetotail or the development of low-frequency waves. Pre-substorm variations of ULF wave parameters were seen not only in geomagnetic, but in optical data as well. Samson et al. (2003) observed FLR in the frequency range $1-4 \mathrm{mHz}$ in optical data from a meridian scanning photometer (MSP) at Rankin Inlet (CGM latitude $\left.\Phi=72^{\circ}\right)$ and Gillam $\left(\Phi=66^{\circ}\right)$ stations. 
A comparison of magnetic and optical pulsations in the $1-4 \mathrm{mHz}$ frequency range could shed light on processes which lead to substorms. Processes in the geomagnetic tail, which may be related to any pre-substorm signatures, are reflected mostly in broadband, high-latitude, long-period ULF fluctuations. Such "geomagnetic noise" requires a quantitative analysis technique, such as that developed by Yagova et al. (2010, 2015). Yagova et al. (2010) found that the spectral content of high-latitude geomagnetic pulsations in the $1-5 \mathrm{mHz}(\mathrm{Pc} 5 / \mathrm{Pi} 3)$ frequency range is characterized by regular diurnal variation and irregular day-to-day variation. The regular fraction is controlled by geomagnetic latitude and magnetic local time (MLT), while the irregular variations of Pc5/Pi3 spectral parameters are controlled by the space weather conditions, both in the solar wind and in the magnetosheath-magnetosphere system. This technique (a description is given in Sect. 2.2) may be especially useful for a comparative analysis of geomagnetic and auroral luminosity pulsations. The power spectral density (PSD) of auroral luminosity pulsations varies together with the constant part of auroral luminosity and depends on the solar zenith angle and on the albedo of the atmosphere. The spectral slope of high-latitude geomagnetic $\mathrm{Pc} 5 / \mathrm{Pi} 3$ pulsations proved to be a factor controlled mostly by the parameters of the magnetic field fluctuations in the magnetosphere-magnetosheath system (Yagova, 2015b). Pc5/Pi3 spectral slope varies in a correlated way within all the high-latitude $\left(\Phi>65^{\circ}\right)$ region at the ground surface, and it is natural to assume that the spectral slope, and more generally, the spectral content of auroral luminosity pulsations at millihertz frequencies are also controlled predominantly by parameters of the magnetosphere and that substorm precursors can be found in spectral parameters of geomagnetic and auroral luminosity pulsations.

In the present study we concentrate on the ULF spectral content in the northern polar cap, using both geomagnetic and auroral luminosity data. The analysis focuses on two datasets, both with similar geomagnetic and solar wind conditions. One dataset contains isolated non-triggered substorms whilst the other does not. The spectral parameters of the two datasets may demonstrate a systematic difference between a magnetically quiet interval followed by a substorm and one not followed by a substorm. If such a parameter ("substorm precursor") does really exist and can be quantified, it may indicate the inner generation mechanism for a non-triggered isolated substorm.

\section{Experimental data and data processing}

\subsection{Observations}

In this study, data from an MSP located at the Kjell Henriksen Observatory $(\mathrm{KHO})$, Svalbard $\left(78.15^{\circ} \mathrm{N}, 16^{\circ} \mathrm{E} ; \Phi=\right.$ $75.12^{\circ}$ ), are used. The MSP has a temporal resolution of $16 \mathrm{~s}$ and an angular resolution of one degree.
IMAGE is a European magnetometer network equipped with three-component flux-gate magnetometers (Tanskanen, 2009). The chain is located approximately along the magnetic meridian 100 (MM100), and it covers CGM latitudes from $\Phi=77$ to $40^{\circ}$. The LYR station is colocated with the KHO. The observational range of the MSP allows ionospheric data coverage at one additional magnetometer site NAL $\left(78.9^{\circ} \mathrm{N}, 11.9^{\circ} \mathrm{E}, \Phi=76.1^{\circ}\right)$. In the present study the data with $10 \mathrm{~s}$ initial time resolution are used. The analysis is undertaken for winter months (from the end of October to first half of March) due to the MSP only operating during the auroral season. The observations cover the years of the minimum (2009-2011) and of the growth phase (2012-2014) of the 24th cycle of solar activity.

\subsection{Data processing}

MSP data are digitized for the location coinciding with the northernmost station of the IMAGE network: NAL. To this end, MSP data are averaged in the band centered at $30^{\circ}$. The width of the band depends on latitude, and it is equal $20^{\circ}$ for $\lambda=557.7 \mathrm{~nm}$ for NAL location. Both the IMAGE magnetometer and MSP data are preliminary low-pass-filtered with the cutoff frequency $f_{h}=6.25 \mathrm{mHz}$ and resampled to the same time resolution of $80 \mathrm{~s}$ (note that $f_{h}$ is the Nyquist frequency for the $80 \mathrm{~s}$ time resolution)

Spectral estimates are carried out in a $1.5 \mathrm{~h}$ sliding window using with the Blackman-Tukey method (Jenkins and Watts, 1968; Kay, 1988) with a 64-point Kaiser window. PSDs, and the magnetic-auroral cross spectra (in both square coherence and phase difference) are calculated for the two horizontal components of the magnetic field and the $557.7 \mathrm{~nm}$ MSP channel. In addition to the cross-spectral analysis, a quantitative description of broad-band pulsations is carried out using the methods described in Yagova et al. (2010, 2015) and the reader is directed to those papers for a more detailed description. Briefly, the technique is based upon an expansion into Legendre polynomials of a log-log spectrum with the resulting coefficients providing a quantitative description of ULF wave activity. To describe pre-substorm variations of Pi3 spectral content, the first three coefficients of the expansion

$\sigma(F) \simeq \sigma_{N}(F)=\sum_{n=0}^{N} L_{n} \overline{P_{n}}(F)$

are used. Here $\overline{P_{n}}$ is the $n$th normalized Legendre polynomial (Korn and Korn, 1968); $\sigma(F)=\log \left(S / S_{0}\right)$ (here $S$ is PSD and $\left.S_{0}=1 \mathrm{nT}^{2} \mathrm{~Hz}^{-1}\right)$ :

$$
\begin{aligned}
& F=\frac{2 F_{U}-\left(F_{\max }+F_{\min }\right)}{F_{\max }-F_{\min }} ; \\
& F_{U}=\log (f), F_{\max }=\log \left(f_{\max }\right), \text { and } F_{\min }=\log \left(f_{\min }\right) .
\end{aligned}
$$

The first three coefficients of Eq. (1) describe the logarithm of PSD, $\sigma$, spectral slope, $\alpha$, and the parameter $Q=$ 
Table 1. Selection criteria for automatic selection of non-triggered substorms (see text for details).

\begin{tabular}{|c|c|c|c|c|c|c|c|c|}
\hline $\begin{array}{l}\text { Variable } \\
f(t)\end{array}$ & $\begin{array}{l}\text { Min } \\
f_{\min }\end{array}$ & $\begin{array}{l}\operatorname{Max} \\
f_{\max }\end{array}$ & $t_{1}$ & erval & $\begin{array}{l}\mathrm{d} f / \mathrm{d} t \text { or } \\
\Delta f(\Delta t)\end{array}$ & Min & Max & $\begin{array}{r}\Delta t \\
\text { for } \mathrm{d} f / \mathrm{d} t \text { or } \delta f\end{array}$ \\
\hline$\Delta B_{X}$, TRO, bay & $80 \mathrm{nT}$ & - & 18 & 24:00 UT & $\left\langle\mathrm{d} B_{x} / \mathrm{d} t\right\rangle$ & $-0.1 \mathrm{nT} \mathrm{s}^{-1}$ & - & $900 \mathrm{~s}$ \\
\hline$\Delta B_{X}$, TRO, backgr. & - & $40 \mathrm{nT}$ & -6 & $-1($ ons $=0)$ & - & - & - & $2 \mathrm{~h}$ \\
\hline Dst & $-50 \mathrm{nT}$ & - & -4 days & 0 & - & - & - & - \\
\hline $\mathrm{IMF}, B_{Z}$ & $-3.5 \mathrm{nT}$ & $5 \mathrm{nT}$ & $-6 h$ & 0 & $\left|\Delta B_{Z}\right|$ & - & $5 \mathrm{nT}$ & $900 \mathrm{~s}$ \\
\hline $\mathrm{IMF}, B_{Y}$ & $-7 \mathrm{nT}$ & $7 \mathrm{nT}$ & $-6 h$ & 0 & $\left|\Delta B_{Y}\right|$ & - & $7 \mathrm{nT}$ & $900 \mathrm{~s}$ \\
\hline$p$ & - & $3 \mathrm{nPa}$ & $-6 h$ & 0 & $|\Delta P|$ & - & $1 \mathrm{nPa}$ & $900 \mathrm{~s}$ \\
\hline $\mathrm{AE}$ & - & $70 \mathrm{nT}$ & $-3 h$ & $-0.5 \mathrm{~h}$ & - & - & - & - \\
\hline
\end{tabular}

$-L_{2}$, which estimates the deviation of the spectrum from the inverse power approximation (color noise) near the central frequency of the interval analyzed.

To reveal specific pre-substorm variations, days with isolated non-triggered substorms at the station TRO $\left(69.66^{\circ} \mathrm{N}\right.$, $18.94^{\circ} \mathrm{E} ; \Phi=66.63^{\circ}$ ) located in the auroral oval are selected and compared with non-substorm days. Parameters used to select substorms meeting non-trigger conditions are summarized in Table 1. The first column of Table 1 gives the parameters and points or region where they have been recorded. The second column gives intervals (minimal and maximal values) allowed for each variable, and the third column shows the time interval for analysis, either in UT (the first line) or in respect to onset time (all the other lines of Table 1). For some parameters not only their absolute values but also the variations are important. They are taken into account either in the form of a time derivative or as the amplitude of variations at some time interval. This information is presented in column 4 . The allowed minimal and maximal values for these parameters and the time intervals used are given in columns 5 and 6 , respectively. As examples, the Dst index should not exceed $-50 \mathrm{nT}$ for 4 days preceding the substorm and the IMF $B_{Z}$ component should vary between -3.5 and $5 \mathrm{nT}$ with a maximum absolute value $\Delta B_{Z}$ at each $15 \mathrm{~min}$ interval of $5 \mathrm{nT}$ (this corresponds to $\partial B_{Z} / \partial t$ of $5.6 \mathrm{pT} \mathrm{s}^{-1}$ ). The last line of the table contains the information on the $\mathrm{AE}$ index of auroral activity used to select isolated substorms, i.e., those that are not influenced by preceding auroral activations. Non-substorm days used for the superposed epoch (SPE) analysis are selected with the same set of IMF and geomagnetic activity parameters as days with non-triggered substorms. Threshold values for the maximum amplitudes of geomagnetic variations are chosen to be the same for the non-substorm days and the pre-substorm hours during substorm days. The selection procedure is applied to intervals from January to March and from October to December between 2005 and 2014.

Days when non-triggered substorms are registered together with the most important selection parameters are given in Table 2. The first three columns of Table 2 give the year, day of the year (DOY), and time $t_{0}$ in hours and min- utes of the substorm onset. This is determined automatically from the TRO $B_{x}$ data as a point when $-\mathrm{d} B_{x} / \mathrm{d} t$ exceeds the threshold value. The fourth column gives the substorm bay amplitude. The fifth column gives the minimal value of IMF $B_{Z}$ during last 6 pre-substorm hours. To control a possible influence of preceding substorms, mean and maximal values of the AE index during the last three pre-substorm hours $\left(\mathrm{AE}_{3}\right)$ are given in columns 6 and 7 . For all the substorms, mean values are well below $100 \mathrm{nT}$; however, local auroral activations with $\mathrm{AE}>100 \mathrm{nT}$ occurred in the evening sector at MM100 for about one half of substorms shown in Table 2. To discriminate the possible post-substorm variations from those developing with an absolutely quiet background, we have selected substorms with $\max \left(\mathrm{AE}_{3}\right)<70 \mathrm{nT}$. Hereafter, we denote them as "non-triggered isolated substorms" and use both groups of non-triggered substorms in SPE analysis. The Akasofu parameter (Akasofu, 1979) has not been used in the selection procedure. It is included in Table 2 to estimate an energy input during hours preceding non-trigger substorms. The last column of the table shows the $6 \mathrm{~h}$ maximal value $\max \left(\varepsilon_{6}\right)$. It is clear from Table 2 that the values for the selected days are typical for quiet days, rather than for classical substorm days, as they lie below the substorm level estimated as $100 \mathrm{GW}$ (Akasofu, 1981; Koskinen and Tanskanen, 2002).

An SPE analysis allowed the ULF parameters (PSD, spectral slope, Q-factor, and square spectral coherence) of the two groups to be compared. Each parameter is averaged over presubstorm intervals on days with non-triggered substorms and for non-substorm days. Zeros of time axes are taken at the time instant $t_{0}$ (Table 2) on substorm days, and identical $t_{0}$ distributions are randomly generated for non-substorm days.

\section{Results of the analysis}

\subsection{Background variations of the oval polar boundary and PSD of geomagnetic and auroral luminosity pulsations}

Both auroral and geomagnetic activity at a given location depends, to a first approximation, on its relative position with 
Table 2. Selected non-triggered substorms. Non-triggered isolated substorms (see text) are given in bold.

\begin{tabular}{|c|c|c|c|c|c|c|c|}
\hline Year & DOY & $t_{0}(\mathrm{hh}: \mathrm{mm})$ & $\Delta B, \mathrm{TRO}, \mathrm{nT}$ & Min IMF $\left(B_{Z}\right), \mathrm{nT}$ & $\operatorname{Mean}\left(\mathrm{AE}_{3}\right), \mathrm{nT}$ & $\operatorname{Max}\left(\mathrm{AE}_{3}\right), \mathrm{nT}$ & $\operatorname{Max}\left(\varepsilon_{6}\right), \mathrm{GW}$ \\
\hline 2005 & 070 & 22:12 & 152 & -1.6 & 28 & 42 & 12 \\
\hline 2009 & 036 & $22: 10$ & 98 & $-\mathbf{3 . 0}$ & 26 & 47 & 14 \\
\hline 2010 & 072 & $20: 10$ & 118 & -2.9 & 84 & 236 & 15 \\
\hline 2010 & 075 & $22: 11$ & 147 & -3.3 & 44 & 111 & 10 \\
\hline 2010 & 300 & 19:31 & 93 & -1.8 & 30 & 44 & 40 \\
\hline 2010 & 319 & $21: 46$ & 262 & -3.5 & 22 & 161 & 38 \\
\hline 2011 & 285 & $19: 40$ & 187 & -3.1 & 72 & 145 & 31 \\
\hline 2011 & 339 & 21:04 & 124 & -2.8 & 27 & 18 & 5.6 \\
\hline 2011 & 351 & $22: 35$ & 149 & -2.7 & 20 & 19 & 8.9 \\
\hline 2012 & 017 & $20: 13$ & 107 & -2.9 & 45 & 82 & 18 \\
\hline 2012 & 303 & 21:47 & 137 & -3.3 & 29 & 26 & 13 \\
\hline 2012 & 313 & 21:46 & 115 & -3.3 & 19 & 22 & 22 \\
\hline 2012 & 315 & $21: 36$ & 248 & -3.5 & 50 & 214 & 18 \\
\hline 2014 & 042 & $22: 00$ & 105 & -3.5 & 43 & 192 & 20 \\
\hline 2014 & 048 & 20:01 & 149 & -2.6 & 41 & 95 & 19 \\
\hline
\end{tabular}

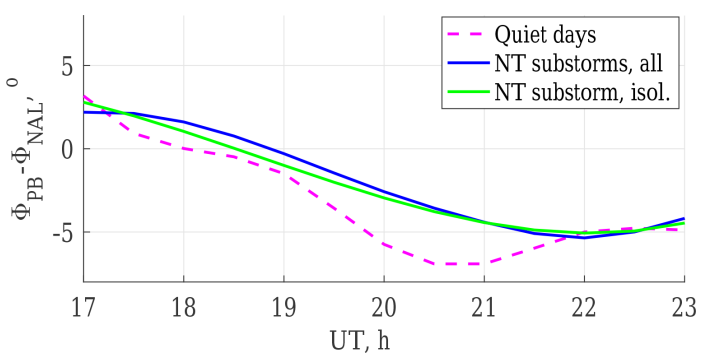

Figure 1. Variations of the difference between the oval polar boundary and the NAL station CGM latitudes, $\Delta \Phi$, averaged over evening and night hours of days with NT substorms (solid blue), isolated NT substorms (solid green), and undisturbed days (dashed magenta).

respect to the auroral oval. The polar boundary of the auroral oval is calculated using DMSP satellite data summarized in the OVATION empirical model (http://sd-www.jhuapl.edu/ Aurora/ovation_live/background.html, Sotirelis et al., 1998; Sotirelis and Newell., 2000). The resulting calculated difference between the auroral polar boundary (PB) and NAL corrected geomagnetic (CGM) latitudes, are shown in Fig. 1. Positive/negative values of $\Delta \Phi$ correspond to the oval/cap NAL position. The variations of $\Delta \Phi$ are shown separately for quiet days (magenta dashed line), days when NT substorms occurred (solid blue line), and isolated non-triggered substorms (green solid line). For all the three datasets NAL crosses the oval PB late in the evening, and it lies inside the polar cap at night. The time of PB crossing is about an hour later for substorm days, than for quiet days, and thus for presubstorm hours PBIs may play a role in observed ULF features.

The diurnal variation of the spectral parameters of geomagnetic and auroral luminosity pulsations averaged over undisturbed days is the background which should be ex- cluded for an analysis of any disturbances in these parameters. Figure 2 illustrates the PSD variations with time in the nighttime MLT sector for pulsations in the geomagnetic (top) and auroral luminosity in the $557.7 \mathrm{~nm}$ channel (bottom). The geomagnetic PSD is calculated in absolute units (PSD is in $\mathrm{nT}^{2} \mathrm{~Hz}^{-1}$ ), while the PSD of auroral luminosity is normalized. The PSD variations for the whole interval of observations are shown with blue solid lines, and the variations for the first (solar minimum) and second (rising phase of solar activity) subintervals are shown with dashed magenta and green dotted lines, respectively. The frequency band centered at $f=1.2 \mathrm{mHz}$ is taken for both geomagnetic and auroral luminosity pulsations. The PSD of the geomagnetic pulsations at 18:00-19:00 UT decreases with time and then grows and reaches a maximum after local magnetic midnight (UT $=21.5$ ). The near-midnight maximum in the geomagnetic PSD is related to substorm-like auroral activity and even for days without substorms the diurnal variation is expressed more clearly for the years 2012-2014 than for the years 2009-2011. Two maxima in the diurnal variation of PSD of auroral luminosity pulsations are found at $\mathrm{UT}=20.3(\mathrm{MLT} \approx 23)$ and $\mathrm{UT}=23.5(\mathrm{MLT} \approx 2.5)$. Thus, the background variations of the PSD of geomagnetic and auroral pulsations are not identical.

Although there are numerous papers describing aurora modulation by Pc5 pulsations (see, e.g., Xu et al., 1993; Safargaleev et al., 2005; Roldugin and Roldugin, 2008), amplitudes of geomagnetic and auroral luminosity pulsations are controlled by different physical factors. Thus, geomagnetic ULF power at high latitudes is essentially controlled by SW or IMF parameters, while the fluctuations of auroral luminosity are more local and their amplitudes depend on the energy distribution of particles at a particular $L$ shell. 


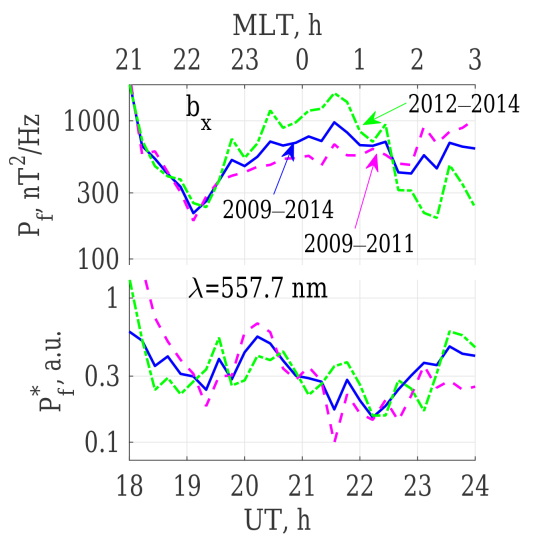

Figure 2. PSD variations of the meridional geomagnetic (top) and $\lambda=557.7 \mathrm{~nm}$ luminosity pulsations (bottom) at nighttime during quiet days at $f=1.2 \mathrm{mHz}$. The results for the whole period of observations (2009-2014), the solar minimum (2009-2011), and the rising phase of solar activity (2012-2014) are shown with blue solid, dashed magenta, and green dotted lines, respectively. PSD of geomagnetic and auroral pulsations are given in $\mathrm{nT}^{2} \mathrm{~Hz}^{-1}$ and in arbitrary units, respectively. UT and MLT for NAL are shown in the bottom and top of the figure.

\subsection{An example of a non-triggered substorm that occurred on day 2011351}

An example of a non-triggered substorm together with the same parameters for an undisturbed day are shown in Fig. 3. The magnetic field variations at the auroral IMAGE station TRO $\left(69.66^{\circ} \mathrm{N}, 18.94^{\circ} \mathrm{E}, \Phi=66.63^{\circ}\right), \mathrm{AE}$ and $\mathrm{Wp}$ indexes (Nose et al., 2012), $B_{Z}$ and $B_{Y}$ IMF components, solar wind velocity, $V_{X}$, and dynamic pressure, $P$, variations are similar for both days. However, a moderate ( $\Delta B \approx 150 \mathrm{nT}$ at TRO) substorm takes place near MLT midnight $(\mathrm{UT}=22: 30)$ on day 2011351 , and no substorm occurs in this longitude sector on day 2009334 . The substorm on day 2011351 is also marked with a peak in Wp index calculated from the lowlatitude Pi2s.

\subsection{Examples of pre-substorm coherent variations of geomagnetic field and auroral luminosity at NAL}

An example of coherent magnetic and auroral luminosity pulsations observed during a $1.5 \mathrm{~h}$ interval starting at 18:40 UT on day 2011351 (in the late evening sector for IMAGE stations) is shown in the left panels of Fig. 4. Broadband pulsations with a visible period about $13 \mathrm{~min}$ are seen simultaneously in the meridional component of the magnetic field at the NAL station and in the intensity of $557.7 \mathrm{~nm}$ emission at the MSP scan angle corresponding to the latitudinal location of NAL. Spectra for this interval are shown in Fig. 5. PSD, square spectral coherence, and phase difference spectra are given in the top, middle, and bottom panels, respectively. Two maxima in the spectral coherence are seen

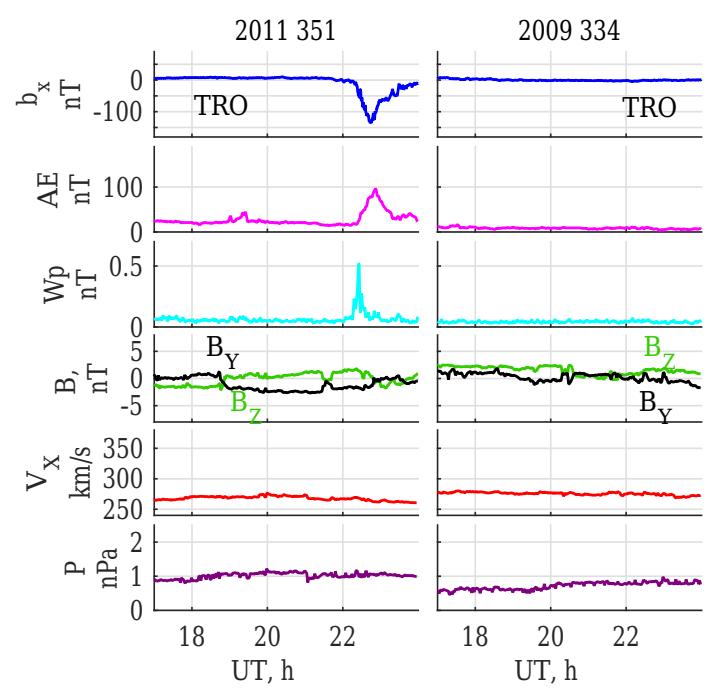

Figure 3. Examples of a "non-triggered" substorm on day 2011351 (left) and a non-substorm on day 2009334 (right). From top to bottom: TRO magnetogram, AE index, Wp index, IMF $B_{Z}$ and $B_{Y}$, solar wind velocity, $V_{X}$, and dynamic pressure, $P$, measured by the ACE and WIND satellites.

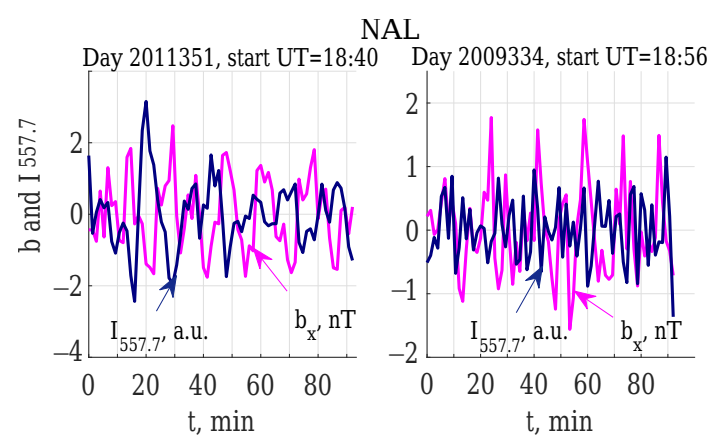

Figure 4. Variations of geomagnetic field and auroral luminosity on days 2011351 (left) and 2009334 (right) shown in Fig. 3 for 1.5 h intervals in the pre-midnight sector. Geomagnetic and auroral luminosity pulsations are given in $\mathrm{nT}$ and arbitrary units, respectively.

at 1.1 and $1.45 \mathrm{mHz}$. As seen from the bottom panel, there is an approximate $180^{\circ}$ phase difference between the pulsations observed in the magnetometer and MSP data across the frequency range with a high coherence level. The substorm starts at 22:30 UT on this day, i.e., $2.5 \mathrm{~h}$ after the end of the interval shown in Fig. 4.

A similar presentation for the interval starting at $\mathrm{UT}=18: 56$ on the non-substorm day 2009334 is given in right panels of Figs. 4 and 5. It is seen from both the time and spectral presentation that magnetic and auroral luminosity pulsations have different spectral content. This is reflected in low spectral coherence (right middle panel of Fig. 5).

Another non-triggered substorm took place on day $2012017\left(t_{0}=20: 13 \mathrm{UT}\right)$. Variations in the magnetic field and auroral luminosity for a $1.5 \mathrm{~h}$ interval starting at 


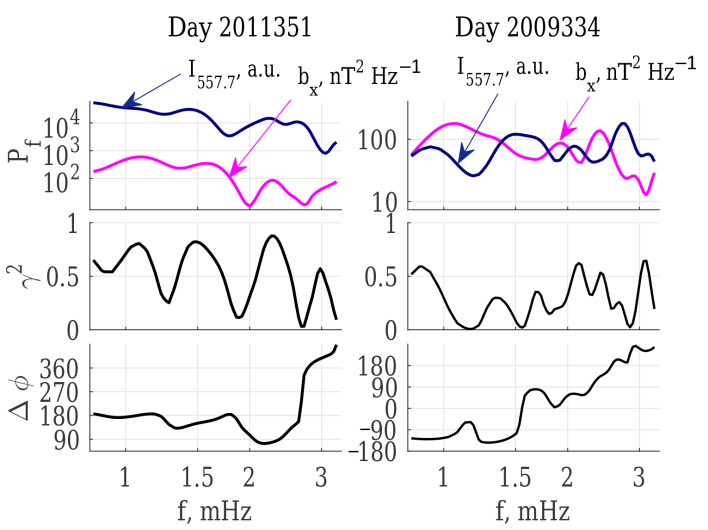

Figure 5. Cross-spectral parameters for geomagnetic and auroral luminosity pulsations for the events shown in Fig. 4. PSD spectra are given in the top panels. The PSD spectra of geomagnetic pulsations (magenta) are given in $\mathrm{nT}^{2} \mathrm{~Hz}^{-1}$, and those of auroral pulsations (dark blue) are given in arbitrary units. Coherence and phase difference spectra are given in the middle and bottom panels, respectively.

Day 2012017, start UT=18:24, NAL

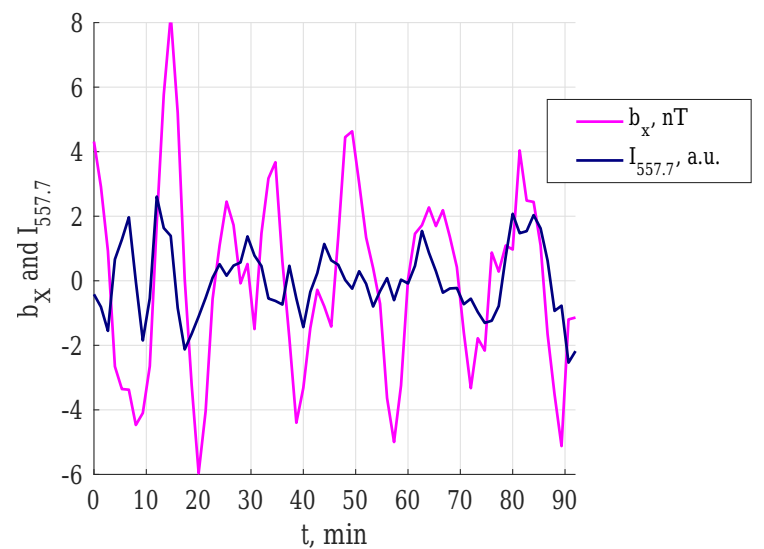

Figure 6. An example of coherent magnetic and luminosity presubstorm pulsations, day 2012017.

18:24 UT are shown in Fig. 6, and the cross-spectral parameters for this event are shown in Fig. 7. Two spectral maxima are seen at approximately 1 and $2.2 \mathrm{mHz}$ in the auroral luminosity and at $1.1 \mathrm{mHz}$ in the geomagnetic pulsations (upper panel of Fig. 7). Also, two maxima are seen in the spectral coherence at 0.9 and $1.8 \mathrm{mHz}$ (middle panel of Fig. 7). At the frequency of the first coherence maximum there is an observed phase shift of approximately $45^{\circ}$ between the auroral and the magnetometer observations (geomagnetic pulsations are leading). By comparison there is no significant phase shift observed at the second coherence maximum (bottom panel of Fig. 7).

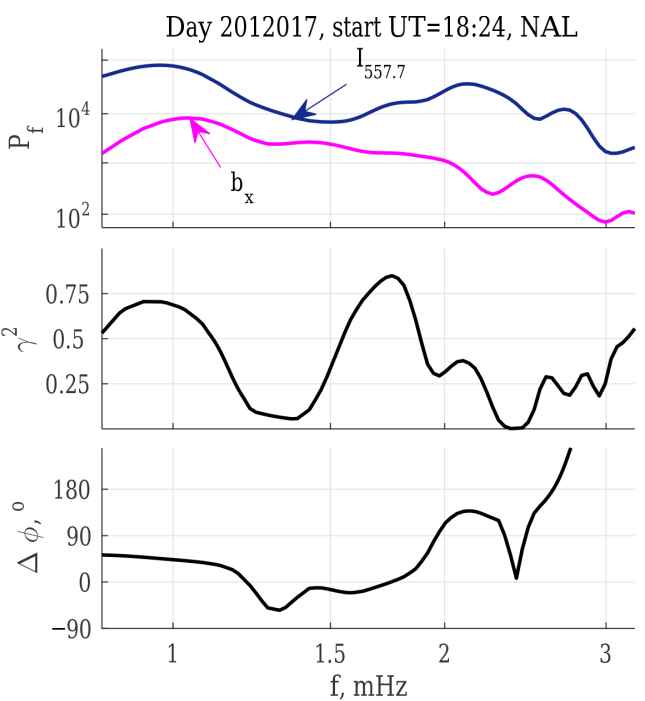

Figure 7. Cross-spectral parameters for geomagnetic (magenta) and auroral luminosity (blue) pulsation for the event shown in Fig. 6. PSD spectra (top), square spectral coherence (middle), and phase difference (bottom). PSD spectrum for the magnetic field is given in $\mathrm{nT}^{2} \mathrm{~Hz}^{-1}$, and auroral luminosity spectrum is given in arbitrary units.

\subsection{SPE results for pre-substorm and non-substorm intervals}

The PSDs at the frequency $f=1.2 \mathrm{mHz}$ averaged over days with non-triggered substorms (solid blue line), and nonsubstorm (dashed magenta line) days are shown in Fig. 8 for the northernmost IMAGE station, NAL, and for the lowerlatitude auroral station TRO for the $15 \mathrm{~h}$ preceding onset time, $t_{0}$, marked as $\tau=0$ in Fig. 8. Note that each point on the time axis shows a starting point of each $90 \mathrm{~min}$ interval, and thus $\tau=-1.5$ corresponds to the last interval before an onset from $t_{0}-1.5$ to $t_{0}$. The same time format is used also in Figs. 9 and 10.

During the entire analysis interval the PSD is almost 1 order of magnitude higher for substorm days than for nonsubstorm days. The situation is qualitatively similar also for the stations BJN $\left(74.5^{\circ} \mathrm{N}, 19.2^{\circ} \mathrm{E} ; \Phi=71.43^{\circ}\right)$ and LYR located between NAL and TRO (not shown). This implies that the amplitude of ULF pulsations in the Pi3/Pc5 range observed at high latitudes preceding a substorm event can be statistically significant when there is no obvious trigger in the SW and pre-substorm conditions at the current point are geomagnetically quiet.

To determine the possible influence of local auroral activations separated by $1-3 \mathrm{~h}$ in MLT, we have selected among non-triggered substorms given in Table 2 those that occurred with a quiet geomagnetic background. To this end, we used the criterion $\max \left(\mathrm{AE}_{3}\right)<70 \mathrm{nT}$. The results for this group of substorms ("non-triggered isolated substorms") are shown in Fig. 8 in green. The behavior of PSD for both groups of 

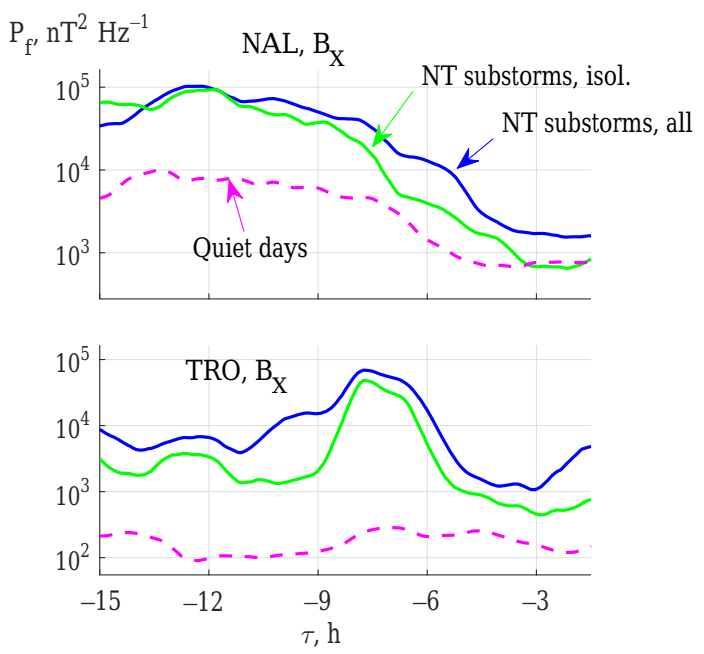

Figure 8. SPE results for PSD at two IMAGE stations averaged over pre-substorm intervals for days with non-triggered substorms (blue), non-triggered isolated substorms (green), and over the intervals with the same MLT distribution for non-substorm days (magenta).

non-triggered substorm days is similar, and it differs essentially from non-substorm days. The difference between the results for all non-triggered substorms and non-triggered isolated substorms is only quantitative, with lower PSD for the non-triggered isolated substorm days.

However, this magnitude difference is evident over more than $10 \mathrm{~h}$ before a substorm onset, and there is no specific change in the PSD which can be utilized to provide information regarding the actual onset time. To reveal specific presubstorm variations, an SPE analysis has also been carried out for the spectral slope, $\alpha$, and the parameter $Q$. The results for the $5 \mathrm{~h}$ preceding substorm onset are shown in Fig. 9. The figure shows a systematic difference between days with non-trigger isolated substorms and non-substorm days during the last pre-onset hours. While for quiet days both $\alpha$ and $Q$ decrease together with PSD, i.e., observed oscillations approach white noise, the situation for the pre-substorm variations of these parameters is different. The spectral slope increases steeply from approximately $t_{0}-4.5 \mathrm{~h}$. A meaningful difference between pre-substorm and quiet spectral slope exists during two time intervals, the first centered at $t_{0}-4 \mathrm{~h}$ and the other starting at $t_{0}-2 \mathrm{~h}$. $Q$ starts growing at about $t_{0}-5$ and reaches a maximum $15 \mathrm{~min}$ later than $\alpha$. A meaningful difference between its pre-substorm and quiet values exists throughout the interval from $t_{0}-4.5$ to $t_{0}-1.5$. This means that $\mathrm{Pi} 3$ spectra are, at that time, dominated by pulsations with frequencies about and below $2 \mathrm{mHz}$.

Correlated pre-substorm variations of the magnetic field and auroral luminosity may indicate the existence of specific pre-substorm pulsations. The SPE results for square spectral coherence, $\gamma^{2}$, between geomagnetic and auroral luminosity pulsations at a central frequency $f=1.4 \mathrm{mHz}$ (i.e., near
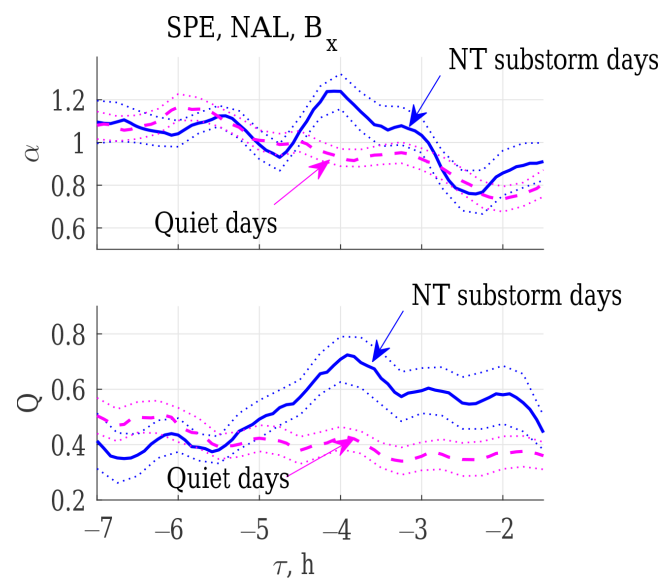

Figure 9. SPE results for spectral slope, $\alpha$, and the parameter $Q$ during the last pre-substorm hours for the same set of non-triggered isolated substorms and non-substorm days as in Fig. 8. Dotted lines show the $85 \%$ confidence intervals.

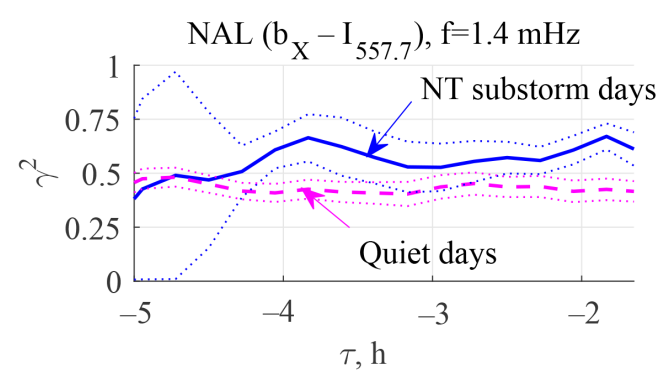

Figure 10. SPE results for the square spectral coherence between auroral $(\lambda=557.7 \mathrm{~nm})$ and magnetic $\left(B_{X}\right)$ pulsations at NAL $(f=$ $1.4 \mathrm{mHz}$ ) for the same days or intervals as in Fig. 8. Dotted lines show the $85 \%$ confidence intervals.

one of the coherence maxima observed in the case studies) are shown in Fig. 10 for the same set of substorm and nonsubstorm days as in Fig. 9. While no essential variation of $\gamma^{2}$ is seen for non-substorm days (dashed magenta line), on substorm days $\gamma^{2}$ demonstrates a maximum at about $t_{0}-3.8 \mathrm{~h}$ and reaches $\gamma^{2}=0.7$. A meaningful difference between presubstorm and non-substorm coherence values exists for two intervals, the first centered at $t_{0}-3.7$ and the second started at $t_{0}-2.2$ when $\gamma^{2}>0.5$. This means that the generation of coherent $\mathrm{Pc} 5 / \mathrm{Pi} 3$ pulsations observed in both the geomagnetic field and auroral luminosity in the polar cap is a characteristic pre-substorm feature, recorded regularly a few hours before an onset of an auroral substorm, or at least before a non-triggered substorm. These pulsations may correspond to some stage of the development of a substorm from various inner processes in the geomagnetic tail. 


\section{Discussion and conclusion}

We have found that the spectral parameters of pulsations observed in the geomagnetic field and auroral luminosity in the polar cap and near the polar boundary of the auroral oval are characterized by both regular background variations and specific pre-substorm changes. A comparison of the ULF pulsation activity in the hours preceding a nontriggered substorm (a substorm that occurs on a geomagnetically quiet day with no discernible trigger in the solar wind) and that observed on a similarly quiet non-substorm day was undertaken. The study found that PSD of geomagnetic fluctuations in the Pc5/Pi3 (1-4 mHz) frequency range is several times higher for these non-triggered substorm days than for non-substorm days. Such an increase in ULF activity could signify favorable conditions for the occurrence of a substorm. Based on precise case studies, Kim et al. (2009) have shown that enhanced levels of IMF fluctuations are related to fluctuating particle flows in the plasma sheet and the enhanced occurrence of onsets in comparison with intervals of non-fluctuating IMF. This result confirms the conclusion of Kozyreva et al. (2007) about the key role that ULF waves observed at high latitudes in the Pc5/Pi3 frequency range play in the energy transfer from the solar wind to the magnetosphere. The basic energy supply channel is reconnection, operated under negative IMF, and viscous interaction, which becomes noticeable under positive IMF. Enhanced ULF turbulence favors the second mechanism (Romanova and Pilipenko, 2008). Additionally, it was proposed that ULF waves can heat the magnetotail plasma and thus provide a condition for the "thermal catastrophe" substorm mechanism (Goertz and Smith, 1989).

ULF power in the outer magnetosphere and at high geomagnetic latitudes on the ground surface is influenced by steep and quasi-periodic variations of solar wind dynamic pressure (Kepko et al., 2002; Kim et al., 2002). Global ULF enhancements following such dramatic changes in solar wind dynamic pressure as a sudden commencement (SC) and sudden impulse (SI) have been studied for almost 50 years, beginning with the early report by Saito and Matsushita (1967). Later the ULF temporal evolution and spatial and frequency distributions of the geomagnetic wave field in the ULF frequency range caused by pressure pulses in the solar wind were studied in numerous papers (see, e.g., Kleimenova et al., 1999, 2011; Roldugin et al., 2006). Yagova et al. (2007) found that ULF spectral power at the high geomagnetic latitudes on the ground correlated with the ULF power in the solar wind at any level with no threshold value of ULF amplitude in the foreshock. Belakhovsky and Roldugin (2008) reported steep variations in ground ULF parameters as an immediate answer to IMF turning without pulses in the solar wind dynamic pressure. An unique Pc5 event registered directly in the geomagnetic tail (Eriksson et al., 2008) also demonstrated a clear answer to the IMF turning. The results of the studies mentioned and numerous others prove that en- hanced ULF activity before triggered, storm time, or nonisolated substorms surely exists, but it can hardly be discriminated from other factors that influence substorm development.

The above analysis shows a contribution of ULF waves to the substorm preparation under the "cleanest" conditions, i.e., when all the non-wave foreshock parameters are fixed at the same level as for non-substorm days. Moreover, there is a principal difference between the preexisting enhanced level of ULF wave power and classical triggers, like the IMF turning or the dynamic pressure pulse. Enhanced ULF amplitude exists for rather a long time before the onset of a nontriggered substorm. Thus, it provides energy input into the magnetosphere but does not choose any particular time instant for the beginning of the process.

We have also found that the spectral content of geomagnetic pulsations at the polar cap station NAL quantitatively described with the spectral slope $\alpha$ and the parameter $Q$ changes during the last $3-4 \mathrm{~h}$ before an onset indicating that the Pi3 spectra are enriched with frequencies below and about $2 \mathrm{mHz}$. Coherent Pc5/Pi3 pulsations recorded in both the geomagnetic field and auroral luminosity in the polar cap are a characteristic pre-substorm feature, observed regularly a few hours before the onset of an auroral substorm or at least before a non-triggered substorm. This effect is evident in both $\mathrm{H}$-component magnetic and $557.7 \mathrm{~nm}$ luminosity pulsations. As for the phase difference between magnetic and auroral luminosity pulsations, it varies from one event to another (see, e.g., Figs. 5 and 7). This effect requires special studies of the magnetic field pulsations in the geomagnetic tail which can be a combination of shear Alfvén and compressional modes and of particle energies responsible for observed auroral luminosity pulsations.

However, the present study leaves several questions open about the preexisting population of ULF waves and their role in the substorm preparation. First of all, at the present stage we cannot conclude that there is no ULF trigger of a "nontriggered substorm" in the interplanetary space, i.e., that a steep change in some of spectral or polarization parameters of the IMF or SW fluctuations does not play the same role as classical substorm triggers.

The next problem which remains open is the nature of the association between the polar cap "precursor" and the auroral substorm. More sophisticated studies are required to understand whether the "substorm precursor" found in the polar cap before a non-triggered substorm is necessarily generated at some stage of a substorm preparation or whether it is related to a forthcoming substorm only statistically. Elucidation of a physical reason for the "substorm precursor" requires the direct comparison of the magnetic field and particle flux variations in several positions including the interplanetary space, the magnetosheath, and the geomagnetic tail. 
Data availability. KHO data are available on request at http://kho. unis.no, and IMAGE magnetometer data are available at http:// space.fmi.fi/image/index.html.

Competing interests. The authors declare that they have no conflict of interest.

Acknowledgements. The authors thank A. K. Sinha for helpful discussions. The IMAGE magnetometer stations NAL and TRO are owned and operated by Troms $\emptyset$ Geophysical Observatory. The Longyearbyen MSP is owned and operated by the University Centre in Svalbard and partly funded by the Research Council of Norway (project number 195385). This research was partly funded by the PolarProg research program under the Research Council of Norway (project number 246725).

The topical editor, Y. Miyoshi, thanks three anonymous referees for help in evaluating this paper.

\section{References}

Akasofu, S. I.: Interplanetary energy flux associated with magnetospheric substorms, Planet. Space Sci., 27, 425-431, 1979.

Akasofu, S.-I.: Energy coupling between the solar wind and the magnetosphere, Space Sci. Rev., 28, 121-190, 1981.

Angelopoulos, V., McFadden, J. P., Larson, D., Carlson, C. W., Mende, S. B., Frey, H., Phan, T., Sibeck, D. G., Glassmeier, K.-H., Auster, U., Donovan, E., Mann, I. R., Rae, I. J., Russell, C. T., Runov, A., Zhou, X.-Z., and Kepko, L.: Tail Reconnection Triggering Substorm Onset, Science, 321, 18653845, doi:10.1126/science.1160495, 2008.

Aubry, M. P., Russell, C. T., and Kivelson, M. G.: On inward motion of the magnetopause before a substorm, J. Geophys. Res., 75, 7018-7031, 1970.

Belakhovsky, V. B. and Roldugin, V. C.: Generation of Pc5 pulsations during the sign reversal of the IMF $\mathrm{Bz}$ component, Geomagn. Aeronomy, 48, 180-186, doi:10.1134/S0016793208020072, 2008.

Eriksson, P. T. I., Blomberg, L. G., Schaefer, S., and Glassmeier, K.-H.: Sunward propagating Pc5 waves observed on the postmidnight magnetospheric flank, Ann. Geophys., 26, 1567-1579, doi:10.5194/angeo-26-1567-2008, 2008.

Gallardo-Lacourt, B., Nishimura, Y., Lyons, L. R., and Donovan, E.: External triggering of substorms identified using modern optical versus geosynchronous particle data, Ann. Geophys., 30, 667673, doi:10.5194/angeo-30-667-2012, 2012.

Goertz, C. K. and Smith, R. A.: The thermal catastrophe model of substorms, J. Geophys. Res., 94, 6581-6596, doi:10.1029/JA094iA06p06581, 1989.

Golovchanskaya, I. V., Kornilov, I. A., and Kornilova, T. A.: Eastwest type precursor activity prior to the auroral onset: Groundbased and THEMIS observations, J. Geophys. Res.-Space, 120, 1109-1123, doi:10.1002/2014JA020081, 2015.

Heacock, R. and Chao, J.: Type Pi Magnetic field pulsations at very high latitudes and their relation to plasma convection in the magnetosphere, J. Geophys. Res., 85, 1203-1213, doi:10.1029/JA085iA03p01203, 1980.
Henderson, M. G.: Auroral Substorms, Poleward Boundary Activations, Auroral Streamers, Omega Bands, and Onset Precursor Activity, in: Auroral Phenomenology and Magnetospheric Processes: Earth And Other Planets, edited by: Keiling, A., Donovan, E., Bagenal, F., and Karlsson, T., American Geophysical Union, Washington, D. C., doi:10.1029/2011GM001165, 2012.

Holter, O., Altman, C., Roux, A., Perraut, S., Pedersen, A., Pecseli, H., Lybekk, B., Trulsen, J., Korth, A., and Kremser, G.: Characterization of low frequency oscillations at substorm breakup, J. Geophys. Res., 100, 19109-19119, doi:10.1029/95JA00990, 1995.

Hsu, T.-S. and McPherron, R. L.: Average characteristics of triggered and nontriggered substorms, J. Geophys. Res., 109, A07208, doi:10.1029/2003JA009933, 2004.

Hurricane, O. A., Fong, B. H., Cowley, S. C., Coroniti, F. V., Kennel, C. F., and Pellat, R.: Substorm detonation, J. Geophys. Res., 104, 10221-10231, doi:10.1029/1999JA900012, 1999.

Jenkins, G. M. and Watts, D. G.: Spectral analysis and its applications, Holden-Day, San-Francisco, Cambridge, London, Amsterdam, 500 pp., 1968.

Kay, S. M.: Modern spectral estimation: Theory and application, Prentice-Hall, Prentice Hall, New Jersey, USA 543 pp., 1988.

Kepko, L., Spence, H. E., and Singer, H. J.: ULF waves in the solar wind as direct drivers of magnetospheric pulsations, Geophys. Res. Lett., 29, 1197, doi:10.1029/2001GL014405, 2002.

Kim, K.-H., Cattell, C. A. , Lee, D.-H., Takahashi, K., Yumoto, K., Shiokawa, K., Mozer, F. S., and Andre, M.: Magnetospheric responses to sudden and quasiperiodical solar wind variations, J. Geophys. Res., 107, 1406, doi:10.1029/2002JA009342, 2002.

Kim, H.-J., Lyons, L. R., Zou, S., Boudouridis, A., Lee, D.Y., Heinselman, C., and McCready, M.: Evidence that solar wind fluctuations substantially affect global convection and substorm occurrence, J. Geophys. Res., 114, A11306, doi:10.1029/2009JA014281, 2009.

Kleimenova, N. G., Kozyreva, O. V., Bitterly, J., and Schott, J.-J.: Geomagnetic Pulsations of the Pc3-5 Range at the Polar-Cusp Latitudes during an SC and Their Global Response, Geomagn. Aeronomy, 39, 428-437, 1999.

Kleimenova, N. G., Kozyreva, O. V., Manninen, J., Raita, T., Kornilova, T. A., and Kornilov, I. A.: High-latitude geomagnetic disturbances during the initial phase of a recurrent magnetic storm (from February 27 to March 2, 2008), Geomagn. Aeronomy, 51, 730-740, doi:10.1134/S0016793211060077, 2011.

Koskinen, H. E. J. and Tanskanen, E.: Magnetospheric energy budget and the epsilon parameter, J. Geophys. Res., 107, 1415, doi:10.1029/2002JA009283, 2002.

Korn, G. A. and Korn, T. M.: Mathematical handbook for scientists and engineers, McGraw-Hill Book Company, New York, San Francisco-Toronto-London-Sydney, 1968.

Kozyreva, O., Pilipenko, V., Engebretson, M. J., Yumoto, K., Watermann, J., and Romanova, N.: In search of a new ULF wave index: Comparison of Pc5 power with dynamics of geostationary relativistic electrons, Planet. Space Sci., 55, 755-769, 2007.

Laundal, K. M. and Østgaard, N.: Persistent global proton aurora caused by high solar wind dynamic pressure, J. Geophys. Res., 113, A08231, doi:10.1029/2008JA013147, 2008.

Liang, J., Liu, W. W., Donovan, E. F., and Spanswick, E.: In-situ observation of ULF wave activities associated with substorm ex- 
pansion phase onset and current disruption, Ann. Geophys., 27, 2191-2204, doi:10.5194/angeo-27-2191-2009, 2009.

Liu, J.-M., Zhang, B.-Ch., Kamide, Y., Wu, Z.-S., Hu, Z.-J., and Yang, H.-G.: Observation of a double-onset substorm during northward interplanetary magnetic field, J. Atmos. Sol.-Terr. Phy., 72, 864-868, doi:10.1016/j.jastp.2010.04.010, 2010.

Liu, J.-M., Kamide, Y., Zhang, B.-C., Hu, H.-Q., and Yang, H.-G.: THEMIS and ground-based observations of successive substorm onsets following a super-long growth phase, Ann. Geophys., 31, 835-843, doi:10.5194/angeo-31-835-2013, 2013.

Lui, A. T. Y.: Revisiting Time History of Events and Macroscale Interactions during Substorms (THEMIS) substorm events implying magnetic reconnection as the substorm trigger, J. Geophys. Res., 116, A03211, doi:10.1029/2010JA016078, 2011.

Lyons, L. R.: A new theory for magnetospheric substorms, J. Geophys. Res., 100, 19069-19081, 1995.

Lyons, L. R.: Geomagnetic disturbances: characteristics of, distinction between types, and relations to interplanetary conditions, J. Atmos. Sol.-Terr. Phy., 62, 1087-1114, 2000.

Mager, P. N. and Klimushkin, D. Yu.: Alfvén ship waves: high$m$ ULF pulsations in the magnetosphere generated by a moving plasma inhomogeneity, Ann. Geophys., 26, 1653-1663, doi:10.5194/angeo-26-1653-2008, 2008.

Newell, P. T. and Liou, K.: Solar wind driving and substorm triggering, J. Geophys. Res., 116, A03229, doi:10.1029/2010JA016139, 2011.

Newell, P. T., Liou, K., Gjerloev, J. W., Sotirelis, T., Wing, S., and Mitchell, E. J.: Substorm probabilities are best predicted from solar wind speed, J. Atmos. Sol.-Terr. Phy., 146, 28-37, 2016.

Nishimura, Y., Lyons, L., Zou, S., Angelopoulos, V., and Mende S.: Substorm triggering by new plasma intrusion: THEMIS all-sky imager observations, J. Geophys. Res., 115, A07222, doi:10.1029/2009JA015166, 2010.

Nishimura, Y., Lyons, L. R., Shiokawa, K., Angelopoulos, V., Donovan, E. F., and Mende, S. B.: Substorm onset and expansion phase intensification precursors seen in polar cap patches and arcs, J. Geophys. Res.-Space, 118, 2034-2042, doi:10.1002/jgra.50279, 2013.

Nose, M., Iyemori, T., Wang, L., Hitchman, A., Matzka, J., Feller, M., Egdorf, S., Gilder, S., Kumasaka, N., Koga, K., Matsumoto, H., Koshiishi, H., Cifuentes-Nava, G., Curto, J. J., Segarra, A., and Celik, C.: Wp index: A new substorm index derived from high-resolution geomagnetic field data at low latitude, Space Weather, 10, S08002, doi:10.1029/2012SW000785, 2012.

Olson, J. V.: Pi2 pulsations and substorm onsets: A review, J. Geophys. Res., 104, 17499-17520, 1999.

Panov, E. V., Sergeev, V. A., Pritchett, P. L., Coroniti, F. V., Nakamura, R., Baumjohann, W., Angelopoulos, V., Auster, H. U., and McFadden, J. P.: Observations of kinetic ballooning/interchange instability signatures in the magnetotail, Geophys. Res. Lett., 39, L08110, doi:10.1029/2012GL051668, 2012.

Pritchett, P. L. and Coroniti, F. V.: A kinetic ballooning/interchange instability in the magnetotail, J. Geophys. Res., 115, A06301, doi:10.1029/2009JA014752, 2010.

Rae, I. J., Murphy, K. R., Watt, C. E. J., Rostoker, G., Rankin, R., Mann, I. R., Hodgson, C. R., Frey, H. U., Degeling, A. W., and Forsyth, C.: Field line resonances as a trigger and a tracer for substorm onset, J. Geophys. Res.-Space, 119, 5343-5363, doi:10.1002/2013JA018889, 2014.
Roldugin, V. K., Maltsev, Y. P., Ostapenko, A. A., and Roldugin, A. V.: Generation of long-period regular pulsations in the magnetosphere during SC: Geomagn. Aeronomy, 46, 438-449, doi:10.1134/S0016793206040050, 2006.

Roldugin, V. C. and Roldugin, A. V.: Pc5 pulsations on the ground, in the magnetosphere, and in the electron precipitation: Event of 19 January 2005, J. Geophys. Res., 113, A04222, doi:10.1029/2007JA012553, 2008.

Romanova, N. and Pilipenko, V.: ULF wave indices to characterize the solar wind - magnetosphere interaction and relativistic electron dynamics, Acta Geophys., 57, 158-170, doi:10.2478/s11600-008-0064-4, 2008.

Safargaleev, V., Sergienko, T., Nilsson, H., Kozlovsky, A., Massetti, S., Osipenko, S., and Kotikov, A.: Combined optical, EISCAT and magnetic observations of the omega bands/Ps6 pulsations and an auroral torch in the late morning hours: a case study, Ann. Geophys., 23, 1821-1838, doi:10.5194/angeo-231821-2005, 2005.

Saito, M. H., Miyashita, Y. , Fujimoto, M., Shinohara, I., Saito, Y., and Mukai, T.: Modes and characteristics of low-frequency MHD waves in the near-Earth magnetotail prior to dipolarization: Fitting method, J. Geophys. Res., 113, A06201, doi:10.1029/2007JA012778, 2008.

Saito, T. and Matsushita, S.: Geomagnetic pulsations associated with sudden commencements and sudden impulses, Planet Space Sci., 15, 573-588, 1967.

Samson, J. C., Wallis, D. D., Hughes, T. J., Creutzberg, F., Ruohoniemi, J. M., and Greenwald, R. A.: Substorm intensifications and field line resonances in the nightside magnetosphere, J. Geophys. Res., 97, 8495-8518, doi:10.1029/91JA03156, 1992.

Samson, J. C., Rankin, R., and Tikhonchuk, V. T.: Optical signatures of auroral arcs produced by field line resonances: comparison with satellite observations and modeling, Ann. Geophys., 21, 933-945, doi:10.5194/angeo-21-933-2003, 2003.

Sotirelis, T. and Newell, P. T.: Boundary-oriented electron precipitation model, J. Geophys. Res., 105, 18655-18673, 2000.

Sotirelis, T., Newell, P. T., and Meng, C.-I.: The shape of the openclosed boundary of the polar cap as determined from observations of precipitating particles by up to four DMSP satellites, J. Geophys. Res., 103, 399-406, 1998.

Tanskanen, E. I.: A comprehensive high-throughput analysis of substorms observed by IMAGE magnetometer network: Years 1993-2003 examined, J. Geophys. Res., 114, A05204, doi:10.1029/2008JA013682, 2009.

Wang, H., Lühr, H., Ma, S. Y., and Ritter, P.: Statistical study of the substorm onset: its dependence on solar wind parameters and solar illumination, Ann. Geophys., 23, 2069-2079, doi:10.5194/angeo-23-2069-2005, 2005.

Wing, S., Gkioulidou, M., Johnson, J. R., Newell, P. T., and Wang, C.-P.: Auroral particle precipitation characterized by the substorm cycle, J. Geophys. Res.-Space, 118, 1022-1039, doi:10.1002/jgra.50160, 2013.

Xu, B.-L., Samson, J. C., Liu, W. W., Creutzberg, F., and Hughes, T. J.: Observations of optical aurora modulated by resonant Alfven waves, J. Geophys. Res., 98, 11531-11541, doi:10.1029/93JA00435, 1993.

Yagova, N., Pilipenko, V., Rodger, A., Papitashvili, V., and Watermann, J.: Long period ULF activity at the polar cap preceding 
substorm, in: Proc. 5th International Conference on Substorms, St. Peterburg, Russia (ESA SP-443), 603-606, 2000.

Yagova, N., Pilipenko, V., Watermann, J., and Yumoto, K.: Control of high latitude geomagnetic fluctuations by interplanetary parameters: the role of suprathermal ions, Ann. Geophys., 25, 1037-1047, doi:10.5194/angeo-25-1037-2007, 2007.

Yagova, N. V., Pilipenko, V. A., Baransky, L. N., and Engebretson, M. J.: Spatial distribution of spectral parameters of high latitude geomagnetic disturbances in the $\mathrm{Pc} 5 / \mathrm{Pi} 3$ frequency range, Ann. Geophys., 28, 1761-1775, doi:10.5194/angeo-281761-2010, 2010.
Yagova, N., Heilig, B., and Fedorov, E.: Pc2-3 geomagnetic pulsations on the ground, in the ionosphere, and in the magnetosphere: MM100, CHAMP, and THEMIS observations, Ann. Geophys., 33, 117-128, doi:10.5194/angeo-33-117-2015, 2015.

Yagova, N. V.: Spectral slope of high-latitude geomagnetic disturbances in the frequency range $1-5 \mathrm{mHz}$. Control parameters inside and outside the magnetosphere, Geomagn. Aeronomy, 55, 32-40, doi:10.1134/S0016793215010144, 2015. 